\title{
Fibrin Clot Delivery System for Meniscal Repair
}

\author{
Suk Hwan Jang, MD, Jeong Ku Ha, MD, Dong Won Lee, MD and Jin Goo Kim, MD, PhD \\ Department of Orthopedic Surgery, Inje University College of Medicine, Seoul, Korea
}

\begin{abstract}
As meniscal preservation particularly in younger active individuals with a symptomatic meniscal tear remains the preferred treatment option, various methods have been suggested to increase healing and success rates after meniscal repair. The recent increase in clinical use of platelet rich plasma has contributed to the increased use of fibrin clot, which virtually has the same healing property. However, despite the relative ease of acquisition and preparation of fibrin clots, delivering it to the desired target area arthroscopically is challenging. Therefore, we report with a pertinent literature review a novel method of planting a fibrin clot to the desired area of meniscal tear arthroscopically using our delivery system to enhance healing.
\end{abstract}

Key words: Meniscus, Meniscus tear, Meniscus repair, Fibrin clot.

With the increasing recognition of the meniscus as an important structure of the knee, meniscal repair has become the preferred treatment of choice over menisectomy ${ }^{1)}$. This is especially true for young and active patients in whom menisectomy has been associated with degenerative arthritis. Longitudinal peripheral tears are an ideal indication for meniscal repair because the peripheral area is rich in blood supply. However, tears that extend into the white-white zone or the white-red zone of the meniscus have been mostly considered irreparable, thus requiring menisectomy. Accordingly, efforts have been made to preserve the knee joint function by repairing tears in the avascular zones ${ }^{2}$. Currently, there is a great deal of interest in platelet rich plasma (PRP) therapy, collagen scaffold implantation, and stem cell therapy that promote tissue regeneration through growth factor release. Accordingly, the use of a fibrin clot that facilitates healing through a similar mechanism has been regaining popularity ${ }^{3)}$.

Received December 30, 2010; Revised (1st) January 25, 2011;

(2nd) April 1, 2011; (3rd) May 7, 2011; Accepted June 7, 2011.

Correspondence to: Jin Goo Kim, MD.

Department of Orthopedic Surgery, Inje University College of Medicine, 85 Jeo-dong 2-ga, Jung-gu, Seoul 100-032, Korea.

Tel: +82-2-2270-0028, Fax: +82-2-2270-0023

Email: boram107@hanmail.net

This is an Open Access article distributed under the terms of the Creative Common Attribution Non-Commercial License (http://creativecommons.org/licenses/by-nc/3.0/) which permits unrestricted non-commercial use, distribution, and reproduction in any medium, provided the original work is properly cited.

Copyright $\odot$ 2011. THE KOREAN KNEE SOCIETY www.jksrr.org
Meniscal repair using a fibrin clot was first introduced by King in 1938 and became popular by Arnoczky and Warren ${ }^{4)}$ in 1983. It has been commonly performed for tears of the inner meniscus where blood supply is poor.

There have been many clinical studies that show good results of meniscal repairs using fibrin clots for the past 20 years. However, there is paucity in the literature regarding the PRP therapy, and the optimal concentration of platelets and growth factors to produce significant physiological improvements over the use of a fibrin clot has not been established. Fibrin clot, which is similar to PRP in terms of the components and healing mechanism, can be prepared with less effort and in a shorter time without expensive equipment and causes few side effects with the use of autologous blood. Thus, it has received considerable attention in the orthopedic field.

However, there are no standardized techniques for meniscal repair using a fibrin clot. Fibrin clots are often destroyed near the arthroscopic portal and can be stuck in the subcutaneous fat layer. In addition, placing a fibrin clot at the target area is challenging because it adheres to sharp edges of surgical instruments such as probes and arthroscopic forceps. For these reasons, surgeons have been reluctant to use fibrin clots.

We report an arthroscopic meniscal repair technique using a fibrin clot designed to successfully deliver fibrin clots to target areas avoiding the abovementioned problems with a review of pertinent literature.

\section{Surgical Technique}

Anteromedial, anterolateral, and superomedial portals were 
created and a diagnostic arthroscopy was performed. The presence of a concomitant injury to the ligament or cartilage was assessed and meniscal tear was identified. When a tear was considered repairable, surgical preparations were made. Unstable fragments and fibrous tissues were removed with a motorized shaver, rasp, or basket forceps and the surface of the meniscus was exposed. The meniscal surface and adjacent synovium were abraded to stimulate vascularization. For the repair of the medial meniscal tears, an arthroscope was inserted via the anteromedial portal and a zone specific cannula was inserted via the anterolateral portal. Vertical mattress sutures using a double arm needle were placed every $4 \mathrm{~mm}$ along the length of the tear in an inside out fashion. An additional suture strand was used when the sutures were not considered strong enough. For the lateral meniscal repairs, an arthrosocpe was inserted through

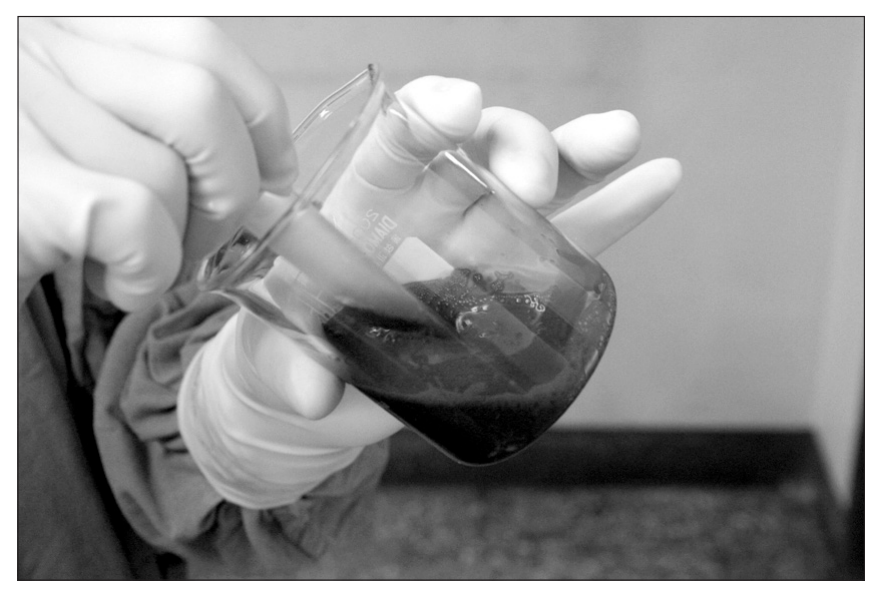

Fig. 1. Autologous blood (50-60 mL) is placed in a sterile $200 \mathrm{~mL}$ beaker and is gently hand stirred with a stir rod for 5 to 10 minutes.
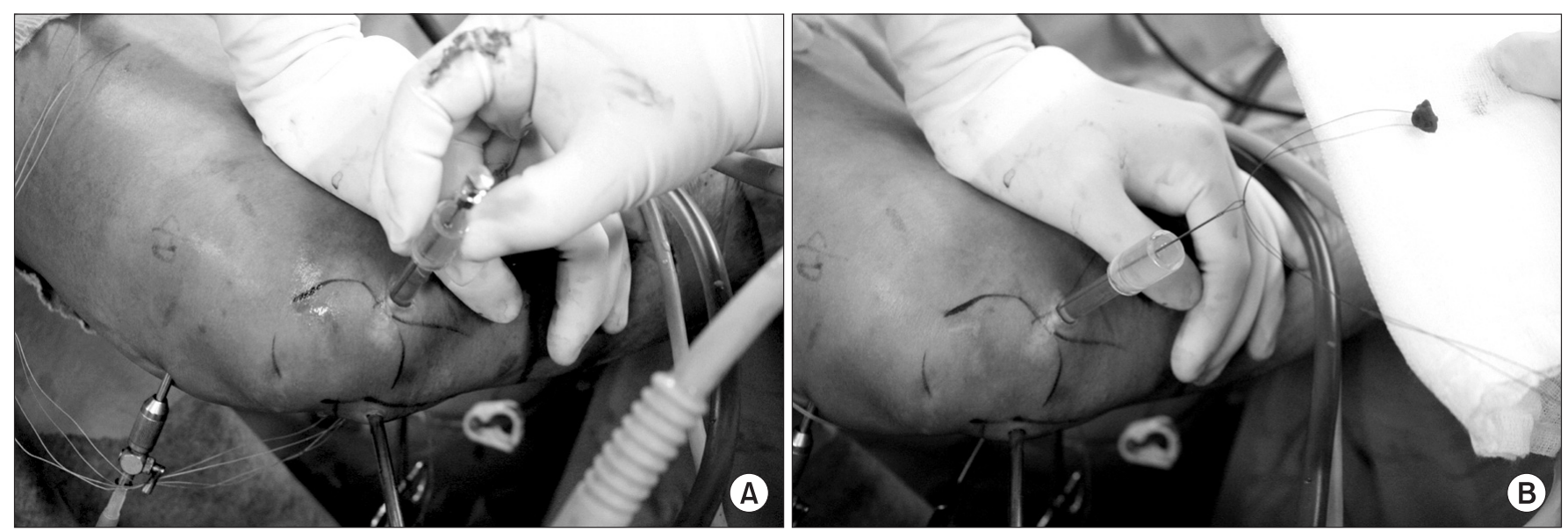

Fig. 3. (A) An eighteen-gauge needle is passed through the plastic cannula to the desired site penetrating the skin. (B) After passing the wire loop through, the 18-gauge needle is removed and the sutured fibrin clot is passed through the loop. the anterolateral portal and sutures were inserted using a zone specific cannula through the anteromedial portal. An accessory incision was made on the medial side for medial tears and lateral side for lateral tears for knot tying and the suture passed through the cartilage was retrieved through it.

Peripheral blood (50-60 mL) was collected from the arm using a sterile syringe and transferred into a $200 \mathrm{~mL}$ beaker (Fig. 1). The blood was gently stirred with a glass rod $(10 \mathrm{~mL}$ glass syringe plunger) for 5 to 10 minutes until 3-5 ml fibrin clot precipitated around the rod. Then, the clot was placed on damp gauze to remove excess fluid and trimmed to proper size (Fig. 2). A transparent shoulder cannula with its polyethylene diaphragm (used to prevent reflux of irrigating fluid) removed to facilitate passage of the fibrin clot was inserted through the anterolateral portal for medial tears and anteromedial portal for lateral tears.

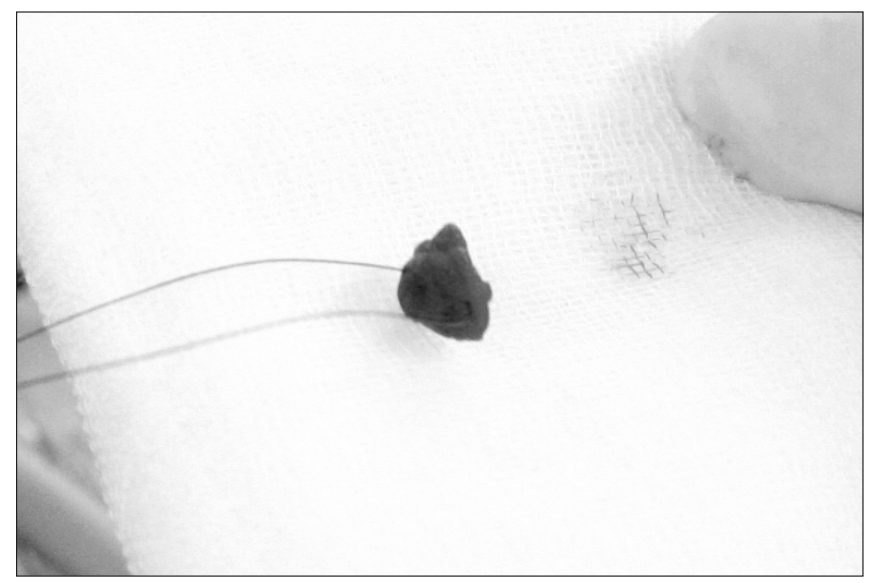

Fig. 2. The fibrin clot is removed from the beaker and placed on sterile gauze. 

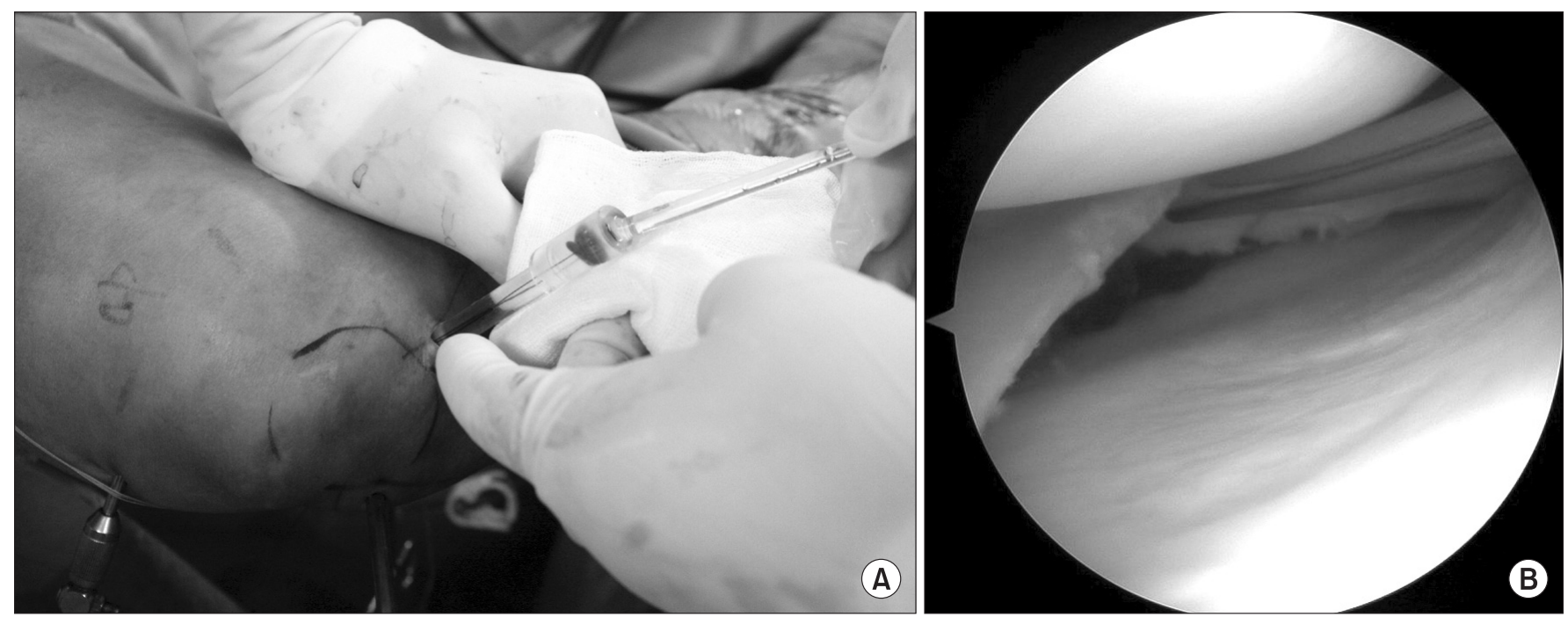

Fig. 4. (A) By gently pulling the suture with the aid of a plastic pusher, the fibrin clot is passed through the plastic cannula. (B) The fibrin clot is planted into the desired intraarticular site.

A $20 \mathrm{~cm}$-long 18-gauge needle was inserted through the cannula, penetrated the target area where the prepared fibrin clot would be added and the skin. A wire loop attached to a needle was inserted through the 18-gauge needle and was withdrawn from the skin, and the outer 18-gauge needle was removed (Fig. 3).

A Nylon no.3 suture was passed through the clot. After removing the suture needle, the suture was passed through the wire loop and pulled out of the skin. Irrigation inflow was turned off to proceed the procedure under a "dry scope." The clot was inserted through the cannula and delivered into the desired intraarticular site by using a plastic pusher and gently pulling the connected suture strand (Fig. 4). After confirming the location of the clot, the sutures that had been pulled through the meniscus out of the joint were ligated.

Partial weight bearing was performed for 6 postoperative weeks in most of the cases. In cases of radial tears, cast immobilization was applied for 3 postoperative weeks, mobilization exercises were started at $0^{\circ}-30^{\circ}$ after the 3 rd postoperative week and advanced gradually until the 6th postoperative week, and partial weight bearing was allowed afterwards.

\section{Discussion}

Between June 2007 and March 2010, 41 meniscal tears (19 radial tears, 12 longitudinal tears in the red-white zone, 7 transverse tears, and 3 oblique tears) were repaired using a fibrin clot at our institution. Second-look arthroscopy or follow-up MRI was performed at an average of 8.3 months after surgery. The success rate of the procedure was $95 \%$ (39 out of 41 ). Once a meniscus is torn or treated with menisectomy, the load-bearing function of the meniscus changes and the knee joint becomes less stable, which leads to a higher risk of osteoarthritis. An in vitro study showed that $16 \%$ to $34 \%$ removal of the meniscus led to a $350 \%$ increase in the load on the articular surface ${ }^{5}$. The meniscus should be preserved whenever feasible especially in young or active patients because they become more vulnerable to osteoarthritis after menisectomy ${ }^{6}$.

However, meniscal tear repairs are not always successful and healing can be delayed or fail. Accordingly, various methods designed to promote healing have been suggested including meniscal rasping, fibrin clot insertion, vascular access channel creation, and synovial flap transfer. The indications for meniscal repair surgery have been extended with use of those procedures that facilitate healing of lesions in the avascular zones ${ }^{2}$.

Webber et al. ${ }^{7)}$ reported that delayed healing was attributable to the absence of a hematoma, not a blood supply per se. A hematoma in a torn meniscus acts as a scaffold that contains growth factors and repair cells for fibrocartilage regeneration through chemotactic activity. The frequency of hematoma formation is lower in the white-white zone or white-red zone compare to the red-red zone.

Arnoczky et al. ${ }^{4)}$ reported that an exogenous fibrin clot acted as a hematoma that promotes healing of the meniscus in dogs. In other words, lesions that had been filled with a fibrin clot healed through a proliferation of fibrous connective tissue that modulated into fibrocartilaginous tissue. Afterwards, many authors have reported on the efficacy of the use of a fibrin clot in clinical settings. In the study of Henning et al. ${ }^{8}$, the failure rate 
of meniscal repair using a fibrin clot was $8 \%$. van Trommel et al. ${ }^{2)}$ reported that all of the 5 lateral meniscus tears involving the popliteal hiatus healed with repair using fibrin clot which were confirmed by second-look arthroscopy. For now, the best clinical results of the fibrin clot use can be expected in isolated meniscal tears.

The recent increase in the use of PRP has led to a high interest in fibrin clots which has similar healing properties that can be obtained without particular equipment at low costs ${ }^{3)}$. However, due to its fragility, delivering the fibrin clot to the exact desired intraarticular site has been considered challenging.

Arthroscopic meniscal repair using a fibrin clot has been the subject of many studies. However, studies that document a delivery method that can be standardized are rare. Although some studies showed that a fibrin clot could be delivered into the joint using a suture or an injection syringe, they are not described in detail and too difficult to reproduce ${ }^{2,8}$. In the study of Sethi et al. ${ }^{9)}$, the synovium near the meniscus was abraded to induce bleeding and the blood ran down and pooled in the torn site to form an in situ fibrin clot. This method is advantageous in that an exogenous fibrin clot delivery method is not needed. However, there is a possibility that the clot may be irrigated away and it is difficult to create a clot in the exact site. van Trommel et al. ${ }^{2)}$ reported successful results of meniscal repair using a fibrin clot, but they did not describe the delivery method that they used. Arthroscopy forceps cannot be helpful in planting fibrin clots in the exact $\operatorname{spot}^{10)}$. A fibrin clot tied with a suture can be delivered from the anterior portal to a desired site, but it is highly likely to be destroyed during the procedure. With our method that has high reproducibility, a fibrin clot can be delivered to a desired site with ease without risk of being damaged by soft tissue during insertion.

Our method can be applied to meniscal tears, especially longitudinal tears in the white-white zone or white-red zone where the likelihood of repair failure is high. In addition, the method is expected to extend the indications for meniscal repair to radial tears where menisectomy is expected to result in functional loss, large-sized transverse tears in young patients, areas between bundles after double-bundle anterior cruciate ligament reconstruction ${ }^{3)}$, and rotator cuff repair. However, we believe a long-term follow-up study should be conducted and biomechanical changes and systemized indications for the use a fibrin clot in meniscal repair should be investigated in future studies.

\section{References}

1. Stein T, Mehling AP, Welsch F, von Eisenhart-Rothe R, Jager A. Long-term outcome after arthroscopic meniscal repair versus arthroscopic partial meniscectomy for traumatic meniscal tears. Am J Sports Med. 2010;38:1542-8.

2. van Trommel MF, Simonian PT, Potter HG, Wickiewicz TL. Arthroscopic meniscal repair with fibrin clot of complete radial tears of the lateral meniscus in the avascular zone. Arthroscopy. 1998;14:360-5.

3. Illingworth KD, Musahl V, Lorenz SGF, Fu FH. Use of fibrin clot in the knee. Oper Tech Orthop. 2010;20:90-7.

4. Arnoczky SP, Warren RF, Spivak JM. Meniscal repair using an exogenous fibrin clot. An experimental study in dogs. J Bone Joint Surg Am. 1988;70:1209-17.

5. Walker PS, Erkman MJ. The role of the menisci in force transmission across the knee. Clin Orthop Relat Res. 1975;(109):184-92.

6. Fairbank TJ. Knee joint changes after meniscectomy. J Bone Joint Surg Br. 1948;30:664-70.

7. Webber RJ, Harris MG, Hough AJ Jr. Cell culture of rabbit meniscal fibrochondrocytes: proliferative and synthetic response to growth factors and ascorbate. J Orthop Res. 1985;3:36-42.

8. Henning CE, Lynch MA, Yearout KM, Vequist SW, Stallbaumer RJ, Decker KA. Arthroscopic meniscal repair using an exogenous fibrin clot. Clin Orthop Relat Res. 1990;(252):64-72.

9. Sethi PM, Cooper A, Jokl P. Technical tips in orthopaedics: meniscal repair with use of an in situ fibrin clot. Arthroscopy. 2003;19:E44.

10. Ha JK, Kim JG, Ra HJ. Meniscus repair with fibrin clot in complete radial tear of lateral meniscus. J Korean Orthop Assoc. 2010;45:92-6. 